\title{
PRODUCT PARTITIONS AND RECURSION FORMULAE
}

\author{
M. V. SUBBARAO
}

Received 30 July 2003

\author{
To my late friend Vincent C. Harris
}

\begin{abstract}
Utilizing a method briefly hinted in the author's paper written in 1991 jointly with V. C. Harris, we derive here a number of unpublished recursion formulae for a variety of product partition functions which we believe have not been considered before in the literature. These include the functions $p^{*}(n ; k, h)$ (which stands for the number of product partitions of $n>1$ into $k$ parts of which $h$ are distinct), and $p_{(d)}^{*}(n ; m)$ (which stands for the number of product partitions of $n$ into exactly $m$ parts with at most $d$ repetitions of any part). We also derive recursion formulae for certain product partition functions without the use of generating functions.
\end{abstract}

2000 Mathematics Subject Classification: 11P81, 11 P82.

1. Introduction. This paper is prompted (and provoked) by a remark made by Kim and Hahn in the introduction of their paper [11] that appeared in this journal a few years back. They said: "we find recursive formulae for the multipartite function $p\left(n_{1}, \ldots, n_{j}\right)$. The most useful formula known to this day for actual evaluation of the multipartite partition function is presented in Theorem 4."

Evidently, they have not noticed the famous paper of Cheema and Motzkin (see [5, Theorem 3.1]) that appeared almost thirty years earlier, wherein Kim and Hahn's result is stated and proved along with some other recursion formulae.

Kim and Hahn also gave in [11, Theorem 6] a product partition version of their [11, Theorem 4], which already appeared in a slightly disguised form in [10, equation 4] of Harris and Subbarao almost nine years earlier. We are not trying to downgrade the importance of the recursive relation for the product partition that Kim and Hahn obtained independently of Harris and Subbarao. For example, it played an important role in Kim's proof that settled an important conjecture of Canfield et al. [1] regarding the distribution of highly factorable numbers. In fact, the author believes that the HarrisSubbarao-Kim-Hahn recursion formula for the product partition functions $p^{*}(n)$ representing the number of product partitions of $n>1$, repetition of parts allowed, is the first recursion formula that appeared in the literature for any product partition function. It is, however, true that a recursive formula for the function $q^{*}(n)$-representing the number of product partitions of $n>1$ into distinct parts-was worked out in the special case when $n$ is square-free by de Bruijn (see [6, Section 6.1, formula (6.1.1)]) in the name of class-partitions of a finite set. Also, in the name of Bell numbers (see, e.g., Carlitz [2]), a recursive relation for $q^{*}(n)$ was known earlier for square-free values of $n$. 
The simple method of obtaining recursion formulae for product partition functions that we describe in Section 3 is applicable to almost all product partition functions. To illustrate this fact, we will derive recursive formulae for a diverse collection of product partition functions in this paper, giving proofs only in a few cases.

In Section 5, we obtain new recursion formulae of a different kind for a certain class of product partitions without using this method, and in fact without any appeal to their generating functions.

2. Some functions and their generators. Recall that by a product (or multiplicative) partition of $n>1$ we mean a representation of $n$ as an unordered product of integers greater than 1 ; the terms in the product are called "parts" of the product partition. Three basic product partition functions are $p^{*}(n), q^{*}(n)$, and $e^{*}(n)$. Here, $p^{*}(n)$ (resp., $q^{*}(n)$ ) denotes the number of product partitions of $n$ with repetition of parts allowed (resp., not allowed). The function $e^{*}(n)$ denotes the excess of the number of product partitions of $n$ into an even number of distinct parts over those into an odd number of such parts. While $p^{*}(n)$ and $q^{*}(n)$ were first introduced into the literature (in a different notation) by MacMahon [12], $e^{*}(n)$ came into the literature only in 2001 (see [14]). Some other product partition functions that we consider here, along with the condition attached to them, are the following:

(i) $p_{(k)}^{*}(n)$ : product partitions of $n$ with no part occurring more than $(k-1)$ times;

(ii) $p^{*}(k)_{(n)}$ : number of product partitions of $n$, where each part can occur in at most $k$ colours;

(iii) $q_{(k)}^{*}(m ; n)$ : number of product partitions of $n$ into exactly $m$ parts, no part repeating more than $(k-1)$ times;

(iv) $p^{*}(k, h ; n)$ : number of product partitions of $n$ into exactly $k$ parts of which exactly $h$ parts are different.

REMARK 2.1. Recursion formulae for the additive analogue of $p_{(k)}^{*}(n)$ and $p_{k}^{*}(m, n)$ were considered by Dutta [7] and Dutta and Debnath [8]. The additive analogue of $p^{*}(k, h ; n)$, but not its recursion formula, was considered by Cheema [4].

The generating function for $p^{*}(n)$ was given by MacMahon [12]—who in fact started the study of product partitions in 1924-as

$$
1+\sum_{n=2}^{\infty} p^{*}(n) n^{-s}=\prod_{n \geq 2}\left(1-n^{-s}\right)^{-1}, \quad \operatorname{Re} s>1 .
$$

We prefer to take this in the following form (in analogy with the generating function for the additive partition function $p(n))$ :

$$
1+\sum_{n=2}^{\infty} p^{*}(n) x^{\ln n}=\prod_{n=2}^{\infty}\left(1-x^{\ln n}\right)^{-1}, \quad 0<x<\frac{1}{e} .
$$

As explained in [10], we obtain (2.2) from (2.1) by a change of variable. Also, (2.2) arises from the observation that $p^{*}(n)$ is the number of solutions of the Diophantine 
equation

$$
x_{2} \ln 2+x_{3} \ln 3+\cdots+x_{n} \ln n=\ln n, \quad n>1 .
$$

As explained in [10], the series in (2.2) is uniformly and absolutely convergent in any closed interval contained in $(0,1 / e)$. Thus, arranging series according to powers of $x$, taking logarithms, differentiating termwise, and equating coefficients of $\ln n$ powers of $x$ can be done.

Similar remarks apply for all the series and products that are involved in the following generating function results (all summations and products, unless stated explicitly otherwise, are from $n=2$ to $\infty$ ):

$$
\begin{aligned}
1+\sum q^{*}(n) x^{\ln n} & =\prod\left(1+x^{\ln n}\right), \\
1+\sum e^{*}(n) x^{\ln n} & =\prod\left(1-x^{\ln n}\right), \\
1+\sum p_{(k)}^{*}(n) x^{\ln n} & =\frac{\prod\left(1-x^{k \ln n}\right)}{\prod\left(1-x^{\ln n}\right)}, \\
1+\sum p^{*(k)}(n) x^{\ln n} & =\prod\left(1-x^{\ln n}\right)^{-k}, \\
1+\sum_{n \geq 2 ; m \geq 1} p_{(k)}^{*}(m ; n) t^{m} x^{\ln n} & =\prod\left(\frac{1-t^{k} x^{k \ln x}}{1-t x^{\ln n}}\right), \\
1+\sum_{\substack{n \geq 2 \\
k \geq 1 \\
0 \leq h \leq k}} p^{*}(k, h ; n) x^{\ln n} z^{k} t^{h} & =\prod\left(1+\frac{x^{\ln n} z}{1-x^{\ln n} z} t\right) .
\end{aligned}
$$

REMARK 2.2. It is of some interest to notice that if $k+1$ is a prime number, then

$$
p_{(k+1)}^{*}(n) \equiv p^{*(k)}(n)(\bmod k+1)
$$

The additive analogue of this is also true, as noticed by Dutta and Debnath [8].

3. Preliminary remarks on product partition function recurrences. As is well known, to every product partition of $n>1$ with canonical representation $n=$ $\prod_{i=1}^{k} p_{i}^{a_{i}}$, there corresponds uniquely a partition of the vector $\bar{a}=\left(a_{1}, \ldots, a_{k}\right)$. Conversely, given a vector partition of $\bar{a}=\left(a_{1}, \ldots, a_{k}\right)$, we can associate with it a product partition of the integer $n=\prod_{i=1}^{k} p_{i}^{a_{i}}$, where the $p_{i}$ 's are arbitrarily chosen distinct primes. We can therefore say that, in general, any given statement concerning vector partitioning of a specified set of vectors has an equivalent statement concerning product partitions of a corresponding set of natural numbers greater than 1. As a simple illustration of this general principle, we quote two theorems due to Cheema [3] and Subbarao [13], respectively.

THEOREM 3.1 [3]. The number of partitions of $\left(n_{1}, n_{2}, \ldots, n_{s}\right)$ into vectors with at least one component odd is equal to the number of partitions of $\left(n_{1}, n_{2}, \ldots, n_{s}\right)$ into distinct parts (vectors). Note that the same result holds if the parts are required to have nonzero components. 
THEOREM 3.2 [13]. The number of product partitions of $n$ into distinct parts equals the number of product partitions of $n$ into parts (repetitions allowed) none of which is a square integer.

Note that Cheema's theorem is an extension of a well-known theorem of Euler that corresponds to the case when $s=1$. Extending this idea, it is in principle possible to obtain a recursion formula for a product partition function $f(n)$ from a given recursion formula for the vector partition analogue of $f(n)$.

Similarly, the reverse of this is in general possible but it gets more and more difficult as the partition function $f(n)$ becomes more and more complicated. Thus, from the recursion formula (3.1.1) of Cheema and Motzkin [5] for $u\left(n_{1}, \ldots, n_{s}\right)$-the number of unrestricted partitions of the vector $\left(n_{1}, \ldots, n_{s}\right)$-namely,

$$
n_{i} u\left(n_{1}, \ldots, n_{s}\right)=\sum_{k_{i} \geq 0}\left(u\left(n_{1}-k_{1}, \ldots, n_{s}-k_{s}\right) \frac{\sum_{t} k_{i}}{t}\right),
$$

the summation on the right-hand side being over all $t$ dividing $\Delta$-the g.c.d. of the set of $k$ 's-it is not too difficult, though not too easy, to derive the recursion formula for the product partition function $p^{*}(n)$ of Harris and Subbarao [10]:

$$
\sum_{d \mid n} \ln \bar{d} p^{*}\left(\frac{n}{d}\right)=p^{*}(n) \ln n
$$

where $\bar{d}=\prod_{i=1}^{\infty} d_{i}, d_{i}=d^{1 / i}$ if this is an integer and $d_{i}=1$ otherwise.

This is actually what Kim and Hahn [11] did for $p^{*}(n)$. The reverse process is also possible of course. But this is not the natural or simplest way to do. The best way to obtain a recursion formula for a given product partition function $g(n)$ in terms of $g(m)$ for $m<n$ is to do so by a direct process without recourse to the recursion formula for the corresponding vector partition function. This is the main purpose of this paper.

We provide here a simple method which we illustrate by deriving recursion formulae for three diverse product partition functions that involve one, two, and three variables, respectively.

\section{Recursion relations}

THEOREM 4.1. Let $d_{i, r}$ be defined, for $i=1,2, \ldots$, as follows:

$$
d_{(i, r)}= \begin{cases}1, & \text { if } d^{1 / i} \text { is not an integer, } \\ d^{1 / i}, & \text { if } d^{1 / i} \text { is an integer and } d \text { is not an } r \text { th power integer } \\ d^{-1 / i}, & \text { if } d^{1 / i} \text { is an integer and } d \text { is an } r \text { th power integer. }\end{cases}
$$

Define

$$
\bar{d}_{(r)}=\prod_{i=1}^{\infty} d_{(i, r)}
$$


Then $p_{(r)}^{*}(n)$ satisfies the recursion

$$
\sum_{d \mid n} \ln \bar{d}_{(r)} p_{(r)}^{*}\left(\frac{n}{d}\right)=p_{(r)}^{*}(n) \ln n
$$

Proof. We have

$$
\prod_{n=2}^{\infty}\left(\frac{1-x^{\ln n^{r}}}{1-x^{\ln n}}\right)=1+\sum_{n=2}^{\infty} p_{(r)}^{*}(n) x^{\ln n} .
$$

Taking logarithms and differentiating with respect to $x$, we have

$$
\begin{aligned}
& \left(\sum_{j=2}^{\infty} \ln j \sum_{i=1}^{\infty} x^{i \ln j}\right)\left(1+\sum_{k=2}^{\infty} p_{(r)}^{*}(k) x^{\ln k}\right)-\left(\sum_{j=2}^{\infty} \ln j \sum_{i=1}^{\infty} x^{i \ln j^{r}}\right)\left(1+\sum_{k=2}^{\infty} p_{(r)}^{*}(k) x^{\ln k}\right) \\
& =\sum_{n=1}^{\infty} p_{r}^{*}(n) \ln n .
\end{aligned}
$$

Call the left-hand side $S_{1}-S_{2}$. Then it is not difficult to see that

$$
\begin{aligned}
& S_{1}=\left(\sum_{n=2}^{\infty} \sum_{\substack{d \mid n \\
d \neq \text { an } r \text { th power integer }}} \ln \bar{d}_{(i, r)}\right)\left(1+\sum_{k=1}^{\infty} p_{(r)}^{*} x^{\ln k}\right), \\
& S_{2}=\left(\sum_{n=2}^{\infty} \sum_{\substack{d \mid n \\
d=r \text { th power integer }}} \ln \bar{d}_{(i, r)}\right)\left(1+\sum_{n=1}^{\infty} p_{(r)}^{*} x^{\ln k}\right) .
\end{aligned}
$$

The result now follows.

REMARK 4.2. For $r=\infty$, the theorem gives the recursion formula for $p^{*}(n)$, and for $r=2$, it gives the recursion for $q^{*}(n)$, namely,

$$
\sum_{d \mid n} \ln \bar{d} q^{*}\left(\frac{n}{d}\right)=q^{*}(n) \ln n,
$$

where

$$
d_{i}= \begin{cases}d^{1 / i}, & \text { if this is an integer and } d \text { is not a square integer, } \\ \bar{d}^{1 / i}, & \text { if this is an integer and } d \text { is a square integer, } \\ 1, & \text { in all other cases. }\end{cases}
$$

This may be compared with its vector partition version given by Cheema and Motzkin (see [5, equation (3.1.2)]). 


\subsection{Recursion formula for $q_{(k)}^{*}(m ; n)$ using its generating function.}

$$
1+\sum_{n \geq 2, m \geq 1} q_{(k)}^{*}(m ; n) t^{m} x^{\ln n}=\prod_{n=2}^{\infty}\left(1-t^{k} x^{k \ln n}\right)\left(1-t x^{\ln n}\right)^{-1} .
$$

Taking logs and differentiating with respect to $x$, we get, after a little simplification,

$$
\sum_{n=2}^{\infty} \sum_{m=1}^{\infty} q_{(k)}^{*}(m ; n) t^{m} x^{\ln n} \ln n=\left(1+\sum_{\substack{m \geq 1 \\ n \geq 2}} q_{(k)}^{*}(m ; n) t^{m} x^{\ln n}\right)(S(1)-S(k)),
$$

where

$$
S(k)=\sum_{i, j=1}^{\infty} k(\ln j) t^{k i} x^{k i \ln j}=\sum_{d}\left(\sum_{i} k d_{i k} t^{i k}\right) x^{\ln d}, \quad d=j^{i k} .
$$

Hence, equating coefficients of $x^{\ln n}$ on both sides of the above equation, we get, after a routine simplification, the recursion formula

$$
\begin{aligned}
(\ln n) q_{(k)}^{*}(m ; n)= & \sum_{d \mid n} \sum_{i \geq 1}\left(\ln d_{i}\right) q^{*}\left(m-i ; \frac{n}{d}\right) \\
& -\sum_{d \mid n} \sum_{i} \ln d_{i k}^{k} q^{*}\left(m-i k, \frac{n}{d}\right),
\end{aligned}
$$

where, as usual, $d_{i}=d^{1 / i}$ if this is an integer and $d_{i}=1$ otherwise.

4.2. Recursion formula for $p^{*}(k, h ; n)$. Starting with the generating function for $f(u, v ; n)$, taking logarithms, and differentiating with respect to $x$, we have, after a routine simplification,

$$
\begin{aligned}
& \sum_{n=2}^{\infty} \sum_{m=1}^{\infty} \sum_{1 \leq v \leq u} \ln n p^{*}(u, v ; n) z^{u} t^{v} x^{\ln n} \\
& =\left\{\frac{d}{d x} \prod_{n=2}^{\infty}\left(1-x^{\ln n} z\right)-\frac{d}{d x} \prod_{n=2}^{\infty}\left(1-x^{\ln n} z(1-t)\right)\right\} \\
& \times\left\{1+\sum_{\substack{n, u, v \\
n \geq 2, u \geq 1 \\
1 \leq v \leq u}} p(u, v ; n) x^{\ln n} z^{u} t^{v}\right\} \\
& =\left(S_{1}-S_{2}\right) T \text {, say. }
\end{aligned}
$$

We want to equate the coefficients of $x^{\ln n} z^{k} t^{h}$ on both sides of this equation. We write

$$
S_{1}=\sum_{j=2}^{\infty} \ln j \sum_{i=1}^{\infty} z^{i} x^{i \ln j}
$$


Write $d=j^{i}$, then $j=d^{1 / i}=d_{i}$, say, where, as usual, $d_{i}=1$ if $d^{1 / i}$ is not an integer and $d_{i}=1$ otherwise.

$$
S_{1}=\sum_{1 \leq i<\infty} z^{i} \ln d_{i} \cdot x^{\ln d}
$$

Remembering that $p^{*}(n, k, h)=0$ if $k<h$, we see that the coefficient of $x^{\ln n} z^{k} t^{h}$ in $S_{1} T$ is

$$
\sum_{d \mid n} \sum_{1 \leq i \leq k-h}\left(\ln d_{i}\right) p^{*}\left(\frac{n}{d}, k-i, h\right)
$$

Note that for $p^{*}(n / d, k-i, h)$ to be unequal to 0 , we should have $k-i \geq h$, that is, $i \leq k-h$.

Similarly, we have

$$
\begin{aligned}
S_{2} & =\sum_{1 \leq i<\infty}\left(\ln d_{i}\right) z^{i}(1-t)^{i} x^{\ln d} \\
& =\sum_{1 \leq i<\infty}\left(\ln d_{i}\right) z^{i}\left(1-\left(\begin{array}{c}
i \\
1
\end{array}\right) t+\left(\begin{array}{c}
i \\
2
\end{array}\right) t^{2}-\cdots \pm(-1)^{i} t^{i}\right) x^{\ln d} .
\end{aligned}
$$

Hence, the coefficient of $x^{\ln d}, x^{k} t^{h}$ in $S_{2} T$ is

$$
\sum_{d \mid n} \sum_{\substack{i \\
1 \leq i \leq k}} \sum_{\substack{1 \leq \ell \leq h \\
0 \leq h-\ell<k-i}}\left(\ln d_{i}\right)(-1)^{\ell}\left(\begin{array}{l}
i \\
\ell
\end{array}\right) p^{*}\left(\frac{n}{d}, k-i, h-\ell\right) .
$$

Hence, the recurrence relation for $p^{*}(k, h ; n)$ is given by

$$
\begin{aligned}
(\ln n) p^{*}(u, v, n)= & \sum_{\substack{d \mid n \\
1 \leq i \leq k-h}}\left(\ln d_{i}\right) p^{*}\left(\frac{n}{d}, k-i, h\right) \\
& -\sum_{\substack{d, i, \ell \\
d \mid n, 1 \leq i \leq k-h \\
0 \leq h-\ell \leq k-i}}\left(\ln d_{i}\right)(-1)^{\ell}\left(\begin{array}{l}
i \\
\ell
\end{array}\right) p^{*}\left(\frac{n}{d}, k-i, h-\ell\right) .
\end{aligned}
$$

5. Recursion formulae without using generating series. Using only the definition and combinatorial arguments, we can obtain recursion formulae for certain product partition functions. We illustrate this by working out the details for the function $f(k ; n)$ defined below.

DEFINITION 5.1. For positive integers $k$ and $n>1, f(k ; n)$ denotes the number of product partitions of $n>1$ into exactly $k$ parts each greater than 1 , repetition of parts being allowed. Further, we define $f(k ; n)=0$ whenever $n$ is not an integer greater than 1 or $k$ is not an integer greater than or equal to 1 .

We also use the following definition. 
DEFINITION 5.2. For natural numbers $a, b$, we write $a \| b$ to mean that $a \mid b$ and g.c.d. $(a, b / a)=1$.

It is easily seen that this implies that if $a^{k} \| b$, then $a^{k+1} \nmid b$.

THEOREM 5.3. For an integer $n>1$ and a divisor $d>1$ of $n$, write

$$
S(d, t)=f\left(k-1 ; \frac{n}{d}\right)+f\left(k-2 ; \frac{n}{d^{2}}\right)+\cdots+f\left(k-t ; \frac{n}{d^{t}}\right)
$$

where $t$ is defined by $d^{t} \| n$. Then

$$
k f(k ; n)=\sum_{1<d \mid n} S(d, t)
$$

Proof. Since each of the $f(k ; n)$ product partitions of $n>1$ contains $k$ parts (which are not necessarily distinct), the total number $T(k ; n)$ of all the parts in all the $f(k ; n)$ partitions is $k f(k ; n)$. We will now obtain $T(k ; n)$ by using a different method of counting the parts in all the $f(k ; n)$ partitions. Namely, first note that any part, say $d$, that occurs in any of the $f(k ; n)$ partitions is (by the definition of product partitions) necessarily a divisor of $n$. Fixing the divisor $d$ of $n$ for a moment, clearly $d$ occurs one or more times in $f(k-1, n / d)$ of these product partitions. Similarly, $d$ occurs two or more times in $f\left(k-2 ; n / d^{2}\right)$ of these product partitions, and so on.

Now, the number of these product partitions under consideration in each of which $d$ occurs exactly once is equal to the number of those product partitions in which $d$ occurs one or more times minus the number of those product partitions in which $d$ occurs two or more times. This means that $d$ occurs exactly once in $f(k-1 ; n / d)-$ $f\left(k-2 ; n / d^{2}\right)$ partitions. Similarly, in each of $f\left(k-2 ; n / d^{2}\right)-f\left(k-3 ; n / d^{3}\right)$ partitions, $d$ occurs exactly two times, and so on. Hence, the total number of times $d$ occurs in all the $f(k ; n)$ partitions is equal to

$$
\begin{gathered}
f\left(k-1 ; \frac{n}{d}\right)-f\left(k-2 ; \frac{n}{d^{2}}\right)+2\left(f\left(k-2 ; \frac{n}{d^{2}}\right)-f\left(k-3 ; \frac{n}{d^{3}}\right)\right)+\cdots \\
=f\left(k-1 ; \frac{n}{d}\right)+f\left(n-2 ; \frac{n}{d^{2}}\right)+\cdots
\end{gathered}
$$

This proves the theorem.

Using a similar procedure, we see that we have a recursive formula for the function $p_{(k)}(m ; n)$-the number of product partitions of $n$ into exactly $k$ parts, no part repeating more than $m$ times. We define $p_{(k)}(m ; n)=0$ if $n$ is not an integer greater than 1 .

THEOREM 5.4. For $n>1, k \geq 1,1<d \mid n$, write

$$
S^{*}(d, u)=\sum_{i=1}^{u} p_{(k-i)}^{*}\left(m ; \frac{n}{d^{i}}\right)
$$


where $u=\min (t, m), t$ being given by $d^{t} \| n$. Then

$$
k p_{(k)}^{*}(m ; n)=\sum_{1<d \mid n} S^{*}(d, u)
$$

REMARK 5.5. In the summation (5.4), we can take $i=1$ to $\infty$, because for $i>u$, the terms in the summation vanish. A similar remark applies to the sum in (5.1).

A NUMERICAL EXAMPLE. We illustrate the use of Theorem 5.3 to evaluate $f(3 ; 60)$. Since 60 can be written as a product of three integers greater than 1 , namely, $15 \cdot 2 \cdot 2$, $10 \cdot 3 \cdot 2,6 \cdot 5 \cdot 2,5 \cdot 4 \cdot 3$, we have $f(3 ; 60)=4$.

The divisors $d>1$ of 60 are $2,3,4,5,6,10,12,15,20,30$, and 60 :

$$
\begin{aligned}
\sum_{1<d \mid 60} S(d, t)= & S(2,2)+S(3,1)+S(4,1)+S(5,2)+S(6,1)+S(10,1)+S(12,1) \\
& +S(15,1)+S(20,1)+S(30,1)+S(60,1) \\
= & 4+2+1+2+1+1+0+1+0+0+0 \\
= & 12=3 f(3,60) .
\end{aligned}
$$

REMARK 5.6. Proceeding as in the proof of Theorem 5.3, we can obtain a recursive formula for the product partition function that represents the number of product partitions of $n$ into $k$ distinct parts greater than 1 , where $k$ is any positive integer. We will not go into details.

REMARK 5.7. H. Gupta [9] considered an additive version of our method for recursive formula for $j$-partite numbers.

\section{Concluding remarks}

6.1. Following the method used in the previous section, it is very easy to show that $e^{*}(n)$ has the recursion formula

$$
\prod_{d \mid n} \bar{d} e^{*}(n \mid d)=n^{-e^{*}(n)},
$$

where, as usual, $\bar{d}=\prod_{i=1}^{\infty} d_{i}, d_{i}=d^{1 / i}$ if $d^{1 / i}$ is an integer and $d_{i}=1$ otherwise. More generally, $p^{*(k)}(n)$ has the recursion formula

$$
k \prod_{d \mid n} \bar{d} p^{*(k)}\left(\frac{n}{d}\right)=n^{(1 / k) p^{*(k)}(n)} .
$$

6.2. The method that we used in Section 5 to obtain recursive formulae without using generating functions is suitable for numerical work in evaluating functions. It has wide applications, especially in deriving recursion formulae of product partitions into a fixed number of parts. We will consider these in a separate paper.

6.3. The Euler pentagonal number theorem shows that the excess of the number of additive partitions of $n$ into an even number of distinct parts over those into an odd number of such parts is bounded-in fact, this excess is $-1,0$, or 1 . 
TABLE 6.1

\begin{tabular}{cccccccccccc}
\hline$k$ & 1 & 2 & 3 & 4 & 5 & 6 & 7 & 8 & 9 & 10 & 20 \\
\hline$f(k)$ & -1 & 0 & 1 & 1 & -2 & -9 & -9 & 50 & 287 & 412 & -981680358 \\
\hline
\end{tabular}

Cheema and Motzkin showed in [5, Theorem 2.I] that for the analogous function in the case of partitions of two-dimensional vectors $\left(n_{1}, n_{2}\right)$, the analogous absolute value is unbounded. We can in fact show that the same is true for the analogous function in the case of vector partitions of $k$-dimensional vectors for every $k \geq 2$. Even more than this follows from the more general theorem (see [14, Theorem 2.9]) which implies that if $f(k)$ denotes the value of $c^{*}\left(p_{1} p_{2} \cdots p_{k}\right)$, where $p_{1}, p_{2}, \ldots, p_{k}$ are distinct arbitrarily chosen primes, then, as $k \rightarrow \infty, \log |f(k)| / k$ is unbounded-and, in fact,

$$
\limsup _{k \rightarrow \infty}\left(\frac{\ln |f(k)|}{k \log k}\right)=1 \text {. }
$$

There are many unsolved problems concerning $e^{*}(n)$ and $p^{*}(n)$ (see $\left.[14,15]\right)$. We content ourselves mentioning only the following.

(I) Is $k=2$ the only value of $k$ for which $f(k)=0$ ?

(II) Are $f(3)=f(4)=1$ and $f(6)=f(7)=-9$ the only cases of $f(k)$ taking a value more than once?

For the convenience of the reader, we give the first few values of $f(k)$ in Table 6.1. Also, $f(30)=17235101634875315375$.

It may be of some interest to know that for $2 \leq n \leq 100$, the function $e^{*}(n)$ takes only the values $-1,0$, or 1 . It takes the value 0 forty times, -1 twenty-eight times, and 1 thirty-one times. The problem of distribution of the values of $e^{*}(n)$ is open. In particular, the density of those $n$ for which $e^{*}(n)=-1,0$, or 1 is an open problem [14].

Yang [15] has proved-as conjectured by Subbarao and Verma [14]-that $f(k)$ changes sign infinitely often and that $|f(k)|$ is not ultimately monotonic, that is, there is no fixed integer $k_{0}$ such that $|f(k)|$ is monotonic for all $k>k_{0}$.

ACKNOWLEDGMENT. This work was supported in part by an NSERC grant.

\section{REFERENCES}

[1] R. Canfield, P. Erdös, and C. Pomerance, On a problem of Oppenheim concerning "factorisatio numerorum", J. Number Theory 17 (1983), no. 1, 1-28.

[2] L. Carlitz, Some remarks on the Bell numbers, Fibonacci Quart. 18 (1980), no. 1, 66-73.

[3] M. S. Cheema, Vector partitions and combinatorial identities, Math. Comp. 18 (1964), 414420.

[4] _ On more restricted partitions, Rocky Mountain J. Math. 3 (1973), 31-34.

[5] M. S. Cheema and T. S. Motzkin, Multipartitions and multipermutations, Combinatorics (Proc. Sympos. Pure Math., Vol. XIX, Univ. California, Los Angeles, Calif., 1968), American Mathematical Society, Rhode Island, 1971, pp. 39-70.

[6] N. G. de Bruijn, Asymptotic Methods in Analysis, 2nd ed., Bibliotheca Mathematica, vol. 4, North-Holland Publishing, Amsterdam, 1961.

[7] M. Dutta, On new partitions of numbers, Bull. Calcutta Math. Soc. 49 (1957), 221-224.

[8] M. Dutta and L. Debnath, On new partitions of numbers. II, Bull. Calcutta Math. Soc. 51 (1959), 77-78. 
[9] H. Gupta, On the partition of J-partite numbers, Proc. Nat. Inst. Sci. India Part A 27 (1961), 579-587.

[10] C. Harris and M. V. Subbarao, On product partitions of integers, Canad. Math. Bull. 34 (1991), no. 4, 474-479.

[11] J. K. Kim and S. G. Hahn, Recursive formulae for the multiplicative partition function, Int. J. Math. Math. Sci. 22 (1999), no. 1, 213-216.

[12] P. C. MacMahon, Dirichlet series and the theory of partitions, Proc. London Math. Soc. (2) 22 (1924), 404-411.

[13] M. V. Subbarao, Product partitions and Euler pairs, Nieuw Arch. Wisk. (4) 15 (1997), no. 3, 207-217.

[14] M. V. Subbarao and A. Verma, Some remarks on a product expansion. An unexplored partition function, Symbolic Computation, Number Theory, Special Functions, Physics and Combinatorics (Gainesville, Fla, 1999) (F. G. Garvan and M. E. H. Ismail, eds.), Dev. Math., vol. 4, Kluwer Academic Publishers, Dordrecht, 2001, pp. 267-283.

[15] Y. Yang, On a multiplicative partition function, Electron. J. Combin. 8 (2001), no. 1, Research Paper 19, 14 pp.

M. V. Subbarao: Department of Mathematical and Statistical Sciences, University of Alberta, Edmonton, Alberta, Canada T6G 2G1

E-mail address: m.v.subbarao@ua1berta.ca 


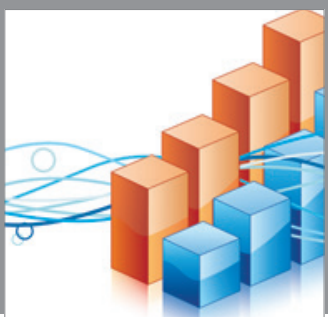

Advances in

Operations Research

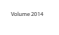

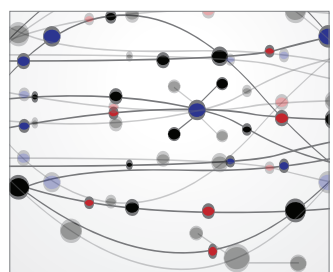

\section{The Scientific} World Journal
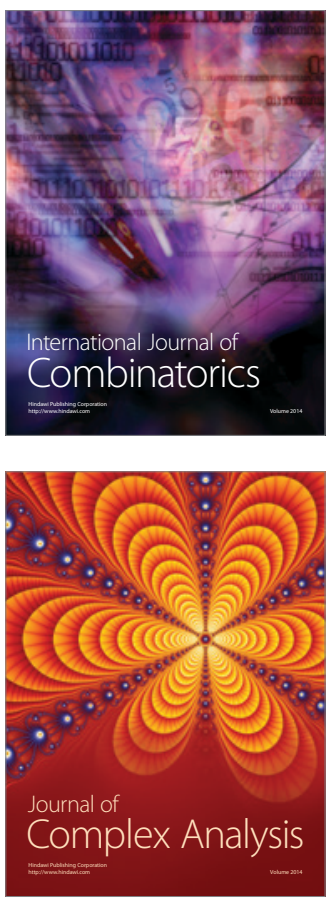

International Journal of

Mathematics and

Mathematical

Sciences
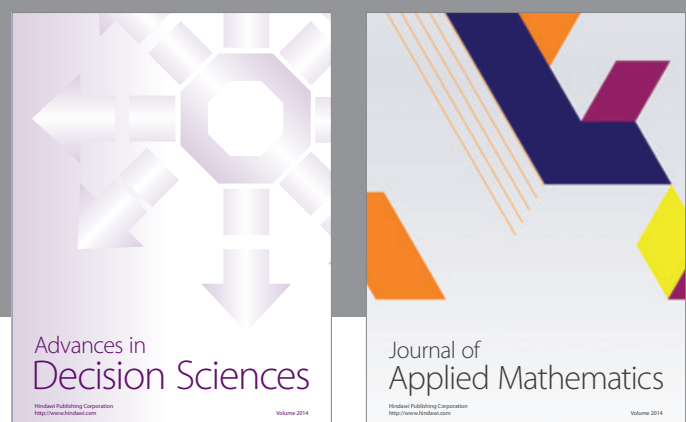

Journal of

Applied Mathematics
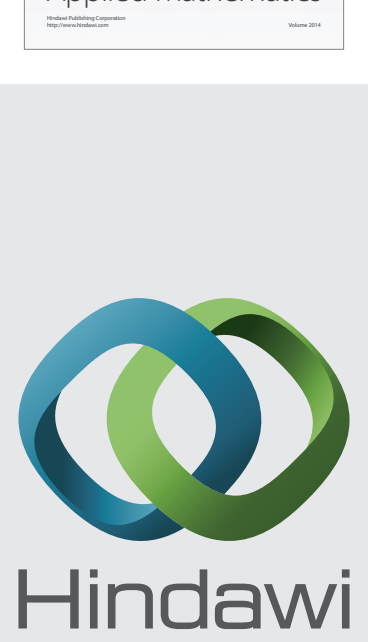

Submit your manuscripts at http://www.hindawi.com
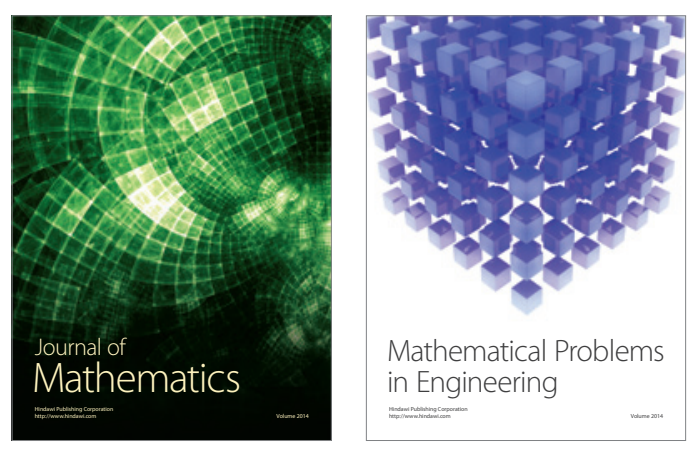

Mathematical Problems in Engineering
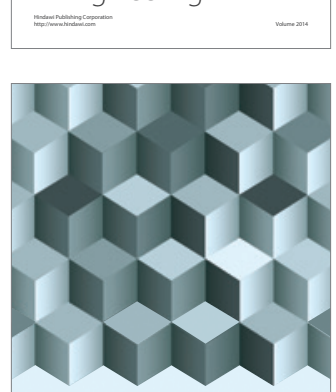

Journal of

Function Spaces
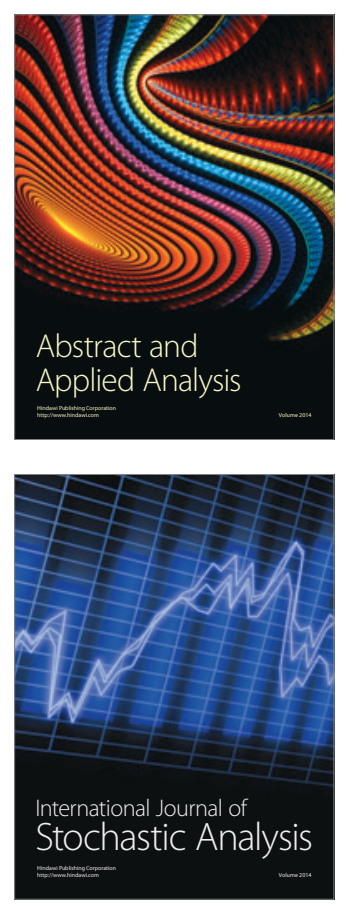

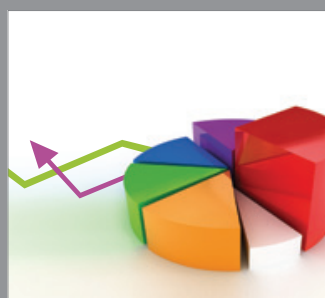

ournal of

Probability and Statistics

Promensencen
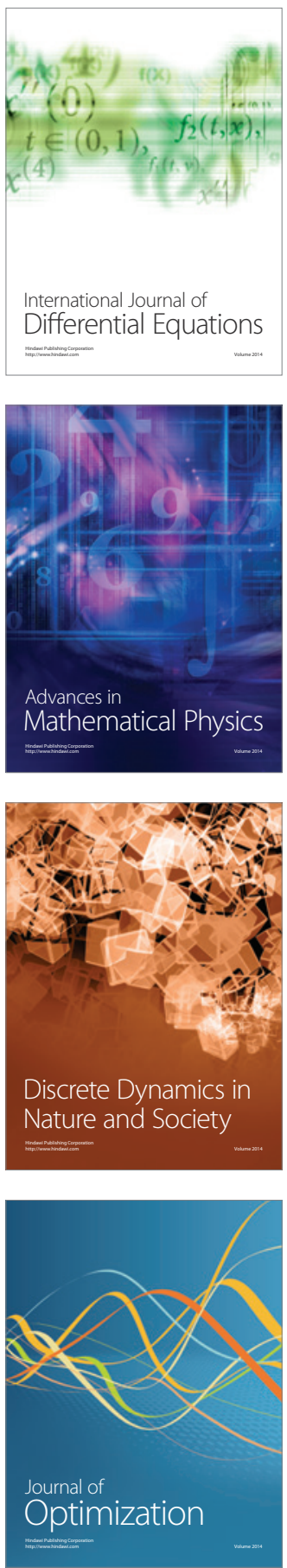